DOI: $10.2478 / \mathrm{cttr}-2013-0880$

\title{
EDITORS' NOTE
}

In this issue of Beiträge, we publish the correspondence on the topic of fibre and particle release from cigarette filters. Dr John L. Pauly (Roswell Park Cancer Institute, NY, USA), protagonist of this research area since the middle of the 1990ies, opened the discussion by sending his views on the article of Hengstberger and Stark [Beitr. Tabakforsch. Int. 23 (2009) 338 - 358] to the Editors. As is customary for scientific journals, we forwarded the letter to the authors and encouraged their public response in Beiträge. We, the Editors, would like to thank Dr Pauly and the authors of the original article for sharing their views with our readers. We also look forward to receiving further correspondence on this and may be other 'hot topics'. 